\title{
Chaillot 1937. Ambitions et réalités d'une recherche architecturale et acoustique
}

Chaillot 1937: Ambitions and Realities of a Research on Architecture and Acoustics

\section{Sandrine Dubouilh}

\section{(2) OpenEdition}

\section{Journals}

Édition électronique

URL : https://journals.openedition.org/rsl/1986

DOI : $10.4000 / \mathrm{rsl} .1986$

ISSN : 2271-6246

Éditeur

Éditions Rue d'Ulm

Référence électronique

Sandrine Dubouilh, «Chaillot 1937. Ambitions et réalités d'une recherche architecturale et

acoustique », Revue Sciences/Lettres [En ligne], 6 | 2019, mis en ligne le 10 décembre 2018, consulté le 30 juillet 2021. URL : http://journals.openedition.org/rsl/1986 ; DOI : https://doi.org/10.4000/rsl.1986

Ce document a été généré automatiquement le 30 juillet 2021.

(c) Revue Sciences/Lettres 


\section{Chaillot 1937. Ambitions et réalités d'une recherche architecturale et acoustique}

Chaillot 1937: Ambitions and Realities of a Research on Architecture and

Acoustics

Sandrine Dubouilh

1 L'objet de cet article est de rendre compte des recherches effectuées sur l'histoire architecturale de la salle de spectacle du Palais de Chaillot, abordée au prisme de sa conception acoustique. Choisie par le programme de recherche ECHO qui s'était organisé autour d'enregistrements sonores de spectacles du TNP (installé dans le bâtiment à partir de 1952), la salle réalisée pour l'Exposition universelle de 1937 est loin d'être exemplaire en matière d'architecture théatrale. Elle succède à la première salle des fêtes, conçue par Gabriel Davioud et Jules Bourdais lors de l'édification du Trocadéro pour l'Exposition universelle de 1878. Elle sera à son tour démolie partiellement en 1975 pour laisser place à une salle expérimentale conçue par Valentin Fabre et Jean Perrottet. Les deux grandes salles de 1878 et 1937 sont liées par leur histoire mais aussi par leur gigantisme, abritant respectivement des jauges de 4665 et 2774 places. Elles sont aussi le produit de commandes institutionnelles étrangères aux nécessités d'un théâtre, ce qu'elles ne sont, de fait, ni l'une ni l'autre. L'acidité des commentaires laissés par les usagers de ces salles, toutes deux disparues, ne pouvait laisser deviner a priori l'intérêt qu'elles recèleraient pour notre étude sur le son du théâtre et sur l'acoustique théâtrale en particulier.

2 Si les effets de la transformation de 1975 sont encore sous nos yeux, c'est bien l'opération de 1937 qui a prioritairement retenu notre attention. Cette configuration frontale, plaçant un vaste gradin surmonté d'un balcon en face de la scène, était un modèle encore peu répandu dans la première moitié $d u x^{e}$ siècle. À partir de 1952, la destination de la grande salle devient majoritairement théâtrale, une activité qui 
n'était pas celle des intentions premières ${ }^{1}$. Ce changement d'usage est en grande partie responsable des écarts entre les ambitions du projet et les réalités de son exploitation.

\section{La salle de l'Exposition universelle}

3 L'histoire du palais du Trocadéro a fait l'objet de peu d'ouvrages en regard de l'importance de ce monument. Parmi ceux-ci, citons celui d'Isabelle Gournay, Le Nouveau Trocadéro, paru en $1985^{2}$, ou plus récemment celui de Pascal Ory, Le Palais de Chaillot, publié en $2006^{3}$. Retraçant l'histoire du bâtiment depuis le premier Palais de 1878, Pascal Ory livre de riches informations sur les salles de spectacle qui y ont trouvé place, valorisant cependant dans ses commentaires et critiques ce que la mémoire collective, dans le sillage des doléances liées à leur utilisation, a enregistré comme deux échecs, le second étant partiellement racheté par l'action du TNP.

Même si le corps central du premier Trocadéro édifié par Gabriel Davioud, architecte, et Jules Bourdais, ingénieur, a disparu en 1937, il n'est pas possible de l'oublier totalement quand on étudie la seconde salle réalisée par Édouard et Jean Niermans, dans les plans du Palais transformé par Jacques Carlu, Louis-Hippolyte Boileau et Léon Azéma. D'un point de vue géométrique, le premier édifice dont les ailes ont été conservées et rhabillées, détermine par ses fondations les limites de la nouvelle salle. Mais plus encore, la rotonde de 1878 fermant la perspective sur la Seine a profondément inspiré son contre-pied, le belvédère prolongé par les jardins, ne laissant d'autre alternative que d'enfouir la grande salle sous la dalle ainsi créée. Ce parti pris radical ne figure cependant pas dans les recommandations du concours pour l'Exposition universelle des «Arts et techniques appliqués à la vie moderne » de 1937. Le plan directeur communiqué aux concurrents prévoit que « la grande salle qui servira de salle d'honneur, des congrès et grandes représentations de galas, devra en outre être entièrement transformée: volumes nouveaux, aménagements modernes de la ventilation et de l'acoustique ${ }^{4} »$. Bien que les limites de cette transformation ne soient pas explicites, il n'est pas question de raser la salle des fêtes pour laquelle le Plan directeur recommande même aux architectes de "ménager des surprises ${ }^{5}$ ». Le programme du concours destiné au «Camouflage du Trocadéro » est assez précis sur ce point :

Sans prévoir de démolition ni de changements notables de la construction existante, les candidats présenteront un projet de décor temporaire masquant complètement les façades actuelles. [...] Son but principal est de changer totalement, pour le temps que durera l'Exposition, l'aspect du Palais, tant du côté des jardins que du côté de la place ${ }^{6}$.

Quelques-unes des réponses à ce concours d'idées qui reçut plus de trois cents projets sont publiées par La Construction moderne du 10 mars 1935. Les esquisses remises jouent abondamment sur les effets d'éclairage, signes de modernité, et de nombreux articles de presse soulignent ce dessein. On comprend mieux pourquoi dans ce concert d'innovations - dont «un immense Planétaire qui mettrait le visiteur au centre du monde tel que le conçoivent actuellement les savants ${ }^{7} »-$, l'ancien Trocadéro avec ses lourdes façades en brique rouge fait tache. Ainsi que le souligne Pascal Ory, «[...] les Expositions universelles sont remplies de ces édifices excentriques et voulus comme tels. Mais avec le monstre - au sens étymologique de ce qui se montre à tous - signé Davioud on a affaire au cas limite d'une architecture qui se donne des airs de rêve 
exotique alors que, passée la fête, [...] on exige d'elle de rentrer dans le rang et de servir $^{8} »$.

6 Cette dimension festive et temporaire n'est pas absente, comme on l'a vu précédemment, du concours de 1937. Le programme du concours de l'Exposition universelle est composé de douze parties indépendantes les unes des autres. Seule la première concerne le Palais du Trocadéro et elle est scindée en deux sections, celle qui concerne le camouflage du bâtiment et celle qui concerne la salle de spectacles. Pour celle-ci, la commande ménage les mêmes incertitudes quant à ce qu'il convient de conserver ou transformer, voire refaire, précisant que «la nouvelle salle devra présenter un volume et un aspect différents de ceux de la salle actuelle ${ }^{9}$ ». Il est dit cependant que le plateau de scène sera «le même » et qu'on conservera à cette "Salle des congrès et d'auditions » une grande capacité d'au moins 6000 places. Un document programme complémentaire reprenant ces éléments, en demandant que la salle soit «complètement modifiée» mais "construite dans l'ancienne avec des moyens simples ", confirme que "le plancher de la scène actuelle subsistera » et destine cet équipement à « des spectacles plus ou moins réduits, des représentations de cinéma, des congrès, des conférences ${ }^{10} \%$. Le moins que l'on puisse dire à la lecture de ces documents officiels, c'est que le cadre de la commande était flou. Mais on comprend surtout qu'il n'y est pas question d'édifier un théâtre, bien que le maintien du Théâtre national populaire, créé en 1921 et installé dans la salle des fêtes du Trocadéro depuis cette date, fasse partie des clauses à prendre en compte.

7 Si l'on considère les opérations de 1878 et 1937, il est évident que les deux aménagements sont marqués par ce contexte très particulier des expositions universelles où se combinent prestige et précipitation. En ce qui concerne le projet de 1937, il n'est pas toujours aisé de comprendre comment se sont déroulées les étapes du glissement du camouflage prévu de la rotonde à la réalisation du belvédère. Mais il est plus difficile encore d'établir avec certitude la chronologie des plans émis par Carlu, Boileau et Azéma d'une part, les frères Niermans d'autre part, pour la réalisation de la salle de spectacles ${ }^{11}$. Le premier monument résultait d'une même série de précipitations et d'imprévus. Initialement envisagé pour être temporaire, le sous-sol de la colline de Chaillot choisie pour accueillir l'édifice se révèlera trop fragile, incitant à la construction d'un bâtiment fondé et en dur. Gabriel Davioud se saisit alors de l'opportunité d'y réaliser, bien que dans un format plus réduit, le projet d'Orphéon sur lequel il travaille depuis le début des années 1860 et pour lequel il a dessiné plusieurs versions. Destiné à l'actuelle place de la République à Paris, alors appelée Place du Château d'eau, ce grand bâtiment devait abriter une salle de concert de 10000 places, sur un plan semblable à celui de la future salle des fêtes, deux fois moins vaste cependant. On notera que cet auditorium, au contraire de la première salle des fêtes du Trocadéro, était doté d'une véritable scène ${ }^{12}$.

\section{Les acousticiens au chevet du Trocadéro}

8 En dépit de l'intérêt porté par Davioud et Bourdais à l'acoustique de la salle des fêtes de $1878^{13}$, celle-ci s'avère rapidement calamiteuse. Son cas nous intéresse pour relater l'intervention successive d'ingénieurs acousticiens à son chevet, initialement dans un but d'amélioration de l'existant, puis d'invention une fois sa démolition décidée. Le premier ingénieur intéressé au sujet est Gustave Lyon, «ancien élève de l'École 
polytechnique, de la maison Pleyel Wolff et $\operatorname{Lyon}^{14}$ ", qui réalisera des mesures et quelques tentatives d'amélioration. L'acoustique défectueuse de la salle des fêtes motive des décisions budgétaires successives, la première en 1904 où un crédit de $78000 \mathrm{~F}$. est voté pour l'amélioration du confort thermique et acoustique de la salle, ceci afin de répondre à la volonté des pouvoirs publics d'y donner des concerts populaires. Quelques années auparavant, l'installation d'un éclairage électrique avait permis d'en allonger l'utilisation, car ainsi que le souligne Jules Bourdais dans son courrier du 6 janvier 1903, la salle des fêtes n'a pu servir « pendant de longues années qu'à des auditions diurnes pendant la saison d'été ${ }^{15}$ ». La question de l'acoustique du Trocadéro est si importante qu'elle motive enfin la création d'une commission spéciale, chargée de suivre les projets d'amélioration. Elle réunit des représentants des services de l'État, des ingénieurs dont Gustave Lyon, et Jules Bourdais désormais désigné comme l'architecte du Trocadéro ${ }^{16}$.

9 La collaboration avec Gustave Lyon a commencé peu avant, sans doute en 1902 ou 1903, celui-ci "prenant à sa charge tous les frais " pour la réalisation "d'instruments nouveaux, construits spécialement en vue de ces expériences ${ }^{17} »$. Celles-ci se sont focalisées sur «l'étude des formes et les natures différentes de diverses parois de la salle, comme aussi sur le degré d'efficacité d'étoffes superposées, et suivant la nature et la position de celles-ci ${ }^{18} »$. De cette première série de mesures, Jules Bourdais conclut cependant qu'il ne croit pas "être en mesure de [...] faire des propositions d'une efficacité certaine", la question étant "très complexe ». Les décisions à prendre ne pouvant émaner du seul duo architecte-ingénieur acousticien, Jules Bourdais préconise donc «de nommer une commission spéciale chargée d'étudier le problème et, si possible, de le résoudre ${ }^{19} »$. Cette commission se réunira régulièrement entre 1904 et 1908, puis, après une interruption consécutive au décès de son président, elle reprendra ses travaux jusqu'en 1912. Les recommandations de Gustave Lyon concernent la pose de panneaux absorbants sur la conque surplombant la scène et l'orgue, Jules Bourdais ayant demandé que le profil de cette voûte concave soit respecté en dépit des difficultés qu'elle générait ${ }^{20}$. La réalisation de ces panneaux de molletons armés n'est pas aisée mais Gustave Lyon est confiant dans leur efficacité dont il espère qu'elle supprimera $90 \%$ des échos ${ }^{21}$. Il est intéressant de noter que Lyon et son équipe n'ont jamais reçu de mission officielle pour ce projet et que le but poursuivi, en marge des activités de la maison Pleyel, Wolff et Lyon $^{22}$, est sans doute en premier lieu scientifique et technique. La salle des fêtes sert de terrain d'expérience pour tester du matériel de mesure et de nouveaux matériaux, ce que l'on peut comprendre par exemple à la lecture d'un rapport de l'Inspecteur général de la $2^{\mathrm{e}}$ division des Bâtiments civils, adressé au Ministre le 16 juin 1906. Dans ce courrier, l'Inspecteur fait bien la part des choses, attirant l'attention du Ministre sur la difficulté de passer des échantillons provisoires de Lyon à une mise en œuvre définitive nécessitant le réglage de nombreux détails techniques et esthétiques: "[...] mais quant à présent il ne s'agit que d'une expérience scientifique: il me parait nécessaire qu'elle soit complètement réalisée avant que la Commission puisse se réunir pour donner un avis sur ce sujet ${ }^{23}$ ». Le traitement de la conque fait l'objet de nombreux désaccords avec Bourdais ${ }^{24}$ mais Gustave Lyon poursuit son travail de conseiller et préconise en 1911 la pose de tentures sur la paroi séparant les tribunes et l'amphithéâtre, ceci devant favoriser l'accueil non seulement des concerts mais aussi des spectacles de l'Opéra-Comique et de la ComédieFrançaise ${ }^{25}$. Cette ultime phase de préconisation, relayée par la Commission de l'acoustique de la salle des fêtes du Trocadéro, prévoit également une transformation 
partielle du plancher de scène pour enfouir l'orchestre et la réalisation « d'un petit théâtre démontable ${ }^{26}$ ", ceci vraisemblablement pour réduire l'espace de la scène en cas de présentation de pièces de théâtre.

10 Si Gustave Lyon se satisfait en 1912 de la correction acoustique qu'il a opérée ${ }^{27}$, la médiocrité de l'écoute dans la salle de spectacle reste unanimement constatée et aggravée par son attribution au (premier) Théatre national populaire, la destinant donc plus encore à la voix parlée, ce pour quoi elle n'avait jamais été conçue. Cette vocation en germe dans les discussions budgétaires de $1903^{28}$ se confirme donc en 1920, attirant une critique sans appel de Pierre Scize parue dans L'Euvre:

Vous allez, Monsieur le Député, fonder un Théatre populaire. Noble entreprise. Courageuse ambition!

Vous allez avoir à votre disposition la nef du Trocadéro. C'est admirable. Vous disposerez là, d'un cirque pouvant contenir cinq mille personnes dont près de deux mille n'entendront à peu près rien, et dont le dernier millier devra s'armer de la lunette méridienne de l'Observatoire pour voir s'agiter sur le lointain plateau d'imperceptibles figurants ${ }^{29}$.

11 Dans les rapports annuels envoyés par Firmin Gémier au ministère de tutelle du Théâtre national populaire, les doléances sur l'inconfort de la salle sont récurrentes. Si le compte-rendu de 1922 suggère de nouvelles améliorations possibles pour l'audition, cette question est cependant mineure en regard des innombrables problèmes que pose l'exploitation de la salle des fêtes du Trocadéro en théâtre.

On comprend mieux cependant à la lumière de cette histoire, pourquoi, bien que vague dans ses préconisations sur la transformation de la salle et les usages auxquels elle sera vouée, le programme de l'Exposition universelle de 1937 ne manque pas de préciser "Étude nouvelle de sa décoration et de son acoustique ${ }^{30}$ ". Cependant, si aujourd'hui un appel à la concurrence pour une salle de spectacle impose aux maîtres d'œuvre de s'associer à un acousticien, il n'en est rien en 1935, année de lancement du concours. L'acousticien qui opèrera sur la salle de spectacle est Jacques Brillouin. L'absence de trace archivistique de son intervention ${ }^{31}$, ainsi qu'une proposition spontanée d'intervention d'un architecte ingénieur conservée dans les archives des bâtiments civils, montrent combien le cadre d'une commande ou d'un marché sur la partie acoustique fait alors défaut. Cette candidature est celle de Robert Fleurent, architecte DPLG (diplômé par le gouvernement), spécialisé en acoustique des salles, en faveur duquel son père, Émile Fleurent, écrit le 22 juillet 1935 au Commissaire général de l'exposition qui a reçu son fils et évoqué «la possibilité de son concours lors de la reconstruction de la salle de théâtre du Trocadéro »:

Depuis qu'il vous a vu il a mis au point des appareils d'une conception nouvelle qui permettront une analyse instantanée des phénomènes sonores. Jusqu'ici les appareils pratiques ne permettaient que des mesures quantitatives c.à.d. de l'intensité des sons ou de leurs composants. Le défaut étant de laisser la plus grande part à l'observateur pour en déduire une appréciation de la qualité sonore. Ces appareils nouveaux éliminent complètement l'opinion des opérateurs; les comparaisons se font soit par une lecture directe soit photographiquement en donnant les indications non seulement de l'intensité sonore mais encore de la qualité même des sons ${ }^{32}$.

13 Ce premier courrier est suivi d'un second en octobre 1935, dans lequel Émile Fleurent « en bon père de famille » insiste sur le fait « qu'il n'existe pas en France d'architecte réellement spécialisé dans les questions acoustiques et à plus forte raison capable de faire par lui-même en pleine connaissance de cause soit les études soit les mesures 
physiques et électriques dont il est désormais impossible de se passer ${ }^{33}$ ". Émile Fleurent poursuit en expliquant qu'ayant appris les noms des lauréats, il a pris contact avec Boileau qu'il connaît, ceci appuyant l'hypothèse qu'à ce stade du concours il n'y avait pas d'acousticien retenu pour participer aux études. La décision de démolir la salle rendit certainement le projet de Robert Fleurent obsolète. On trouve cependant trace de son invention dans un des comptes-rendus hebdomadaires de l'Académie des sciences, où, lors de la séance du 21 mars 1938, Charles Fabry présente « [1]a mesure du temps de réverbération et des niveaux acoustiques moyens à l'aide du fluxmètre. Note de MM. Robert Fleurent et Maurice Beauvilain », en des termes plus scientifiques que ceux utilisés par le père de l'architecte trois ans plus tôt. Le principe de ces appareils de mesure est d'améliorer la précision des appareils existants, utilisant jusqu'alors des galvanomètres balistiques. Outre les avantages du fluxmètre, cet article précise que Fleurent et Beauvilain ont également «mesuré les coefficients d'absorption apparents de quelques matériaux absorbants, soit par des mesures de temps de réverbération, soit par des mesures de niveaux acoustiques moyens ${ }^{34} »$. Robert Fleurent poursuivit sa vocation de spécialiste en acoustique ainsi qu'en témoigne un article paru dans L'Architecture d'aujourd'hui en juin 1949 intitulé "Le laboratoire de recherches et d'essais acoustiques », puis la publication d'une conférence donnée le 25 mai 1954 sur "l'insonorisation des bureaux ${ }^{35}$ ».

Il n'est pas possible en l'état de nos recherches d'expliquer quand et comment Jacques Brillouin fut sollicité pour étudier l'acoustique de la salle non plus remodelée dans l'existant mais bien reconstruite sous la dalle du Trocadéro. Une chose est certaine, le projet acoustique si particulier de la nouvelle salle n'aurait pas existé sans le maintien de l'orgue, construit en 1878 par Aristide Cavaillé-Coll.

\section{Une salle de concert à l'acoustique modulable}

En effet, si de récents projets de salles de concert, notamment le Centre de culture de Lucerne (KKL) et la Philharmonie de Paris ${ }^{36}$, ont mis en œuvre des volumes couplés, autrement dit la possibilité d'augmenter le volume acoustique de la salle et la réverbération par des réserves d'espace ouvertes ou non sur la salle en fonction des besoins, on trouve cette disposition dès 1937 à Chaillot. La création de chambres de réverbération, qu'on trouve fréquemment nommées " chambres d'écho ", est rapportée par Le Génie civil, dans l'article consacré à cette opération le 21 mai $1938^{37}$, mais elles étaient tombées dans l'oubli, sans doute en raison de leur désuétude. Ces chambres de réverbération sont au nombre de trois, deux d'entre elles sont placées de part et d'autre de l'auditorium dans l'espace résiduel résultant de l'inscription du trapèze des gradins dans l'enveloppe rectangulaire de la salle. La troisième est située au-dessus du cadre de scène. Le Génie civil en détaille le fonctionnement : les chambres sont invisibles pour le public, masquées par la continuité du matériau de parement des parois latérales en staff perforé. Des volets pivotants, dont l'ouverture ou la fermeture peut être commandée au pupitre par le chef d'orchestre, présentent deux faces, l'une absorbante, l'autre réfléchissante, ceci permettant de moduler les caractéristiques acoustiques de la salle en fonction des spectacles. Dans un court article paru dans La Revue musicale en 1937, Jacques Brillouin expose clairement le parti pris acoustique comme étant celui d'une salle de concert : 
La salle de spectacle du nouveau Trocadéro a été conçue essentiellement comme une salle d'audition. Il faut s'en féliciter car Paris ne manque pas d'excellents théâtres mais est assez pauvre en salles de concert. Ainsi, l'on voit constamment des grandes manifestations musicales se dérouler dans les théâtres, et parfois même dans les églises, lorsque l'orgue y joue un rôle important ${ }^{38}$. impératifs sur toute la composition, est bien, sous la plume de Jacques Brillouin, la musique, comme en témoigne la suite de l'article :

Cependant, l'expérience ayant montré qu'une salle de cette importance devait pouvoir être utilisée également pour des spectacles, une vaste scène a été réalisée. Cette double destination entraînait de grosses difficultés pour la réalisation d'une acoustique correcte. La musique restant la destination principale de la salle, il ne s'agissait plus comme à l'ordinaire d'aménager tant bien que mal une salle de spectacle. On devait logiquement partir de la salle de concert et y ajouter une scène lorsque le besoin s'en ferait sentir ${ }^{39}$.

Pour imaginer ces chambres de réverbération, Jacques Brillouin explique avoir pris exemple sur les vases d'airain évoqués par Vitruve dans ses Dix livres d'architecture :

[...] la musique exige d'une salle une sonorité plus ronde, plus nourrie que la parole. Nous nous sommes préoccupés de ce point. Grâce à l'utilisation de vastes locaux vides situés sur les côté [sic] de la salle et au-dessus du cadre de scène, nous avons pu réaliser un dispositif propre à augmenter la sonorité de la salle lorsqu'on y exécutera de la musique. Ce procédé est inspiré de la méthode employée par les anciens dans leurs théâtres, et qui consistait à disposer au voisinage de la scène et en divers points de l'amphithéâtre d'énormes vases résonants. La description qu'en donne Vitruve ne laisse aucun doute sur l'effet de ces vases : ils donnaient aux voix la rondeur et la résonance qui leur manque habituellement en plein $\operatorname{air}^{40}$.

Or, s'il est un instrument qui exige une grande réverbération, c'est bien l'orgue. Celui du Trocadéro, installé dès 1878 et qui restera en place jusqu'en 1972 est composé de 4500 tuyaux dont les plus grands mesurent près de dix mètres de haut, plus exactement trente-deux pieds, et pèsent $250 \mathrm{~kg}$ chacun. Le devenir de cet immense instrument en 1937 dans la nouvelle salle est un sujet épineux, mais les problèmes posés par son entretien sont déjà un sujet récurrent dans l'histoire de la première salle des fêtes. Sa dégradation avait notamment suscité l'initiative de l'organiste Marcel Dupré en 1927, lançant une souscription afin de collecter des fonds permettant sa révision, ce qui fut fait. Pour l'opération de 1937, Marcel Dupré est consulté et demande à être accompagné de deux autres organistes, Édouard Mignan et Ludovic Panel. Au final, une commission de cinq organistes sera chargée d'examiner les propositions de transformation de l'orgue faisant par ailleurs l'objet d'un concours auprès des facteurs d'instruments ${ }^{41}$. En effet, dans un courrier adressé à Jacques Carlu le 24 juillet 1935, l'entreprise Cavaillé-Coll recommandait déjà de "profiter du démontage pour le moderniser », en transformant la "traction mécanique en traction pneumatique ${ }^{42}$ ». Mais la transformation envisagée par les architectes va plus loin. Une série de plans de 1935 des architectes Carlu, Boileau et Azéma montre clairement le projet de scinder l'orgue en trois parties distinctes: les grands tuyaux devant prendre place de part et d'autre du cadre, les autres étant installés sur la scène. Cette hypothèse est repoussée par la commission des organistes, expliquant les conséquences d'un tel choix, notamment la nécessité d'électrifier l'orgue. Un courrier de Ludovic Panel à Carlu, daté du 2 août 1936, rappelle quant à lui que la commission refusera toute disposition ayant pour effet de masquer l'orgue, son utilisation optimale exigeant qu'il soit « replacé en 
fronton et parlant directement dans la salle sans être masqué ni assourdi par quoi que ce soit ${ }^{43} »$.

Trouver la place d'un tel monument dans la nouvelle salle ne va donc pas de soi. La transformation de l'orgue est finalement confiée à Fernand Gonzales. Dans le même numéro de La Revue musicale, il livre un bref aperçu des problèmes posés par cet instrument dans la salle de spectacle, mettant en évidence les inventions techniques motivées par la polyvalence du lieu. En effet, dès lors qu'il fut admis que l'emplacement le plus favorable pour cet instrument était la scène elle-même, restait à trouver un dispositif permettant de faire varier son emplacement sur le plateau en fonction des types de spectacle. Fernand Gonzalez rend ici hommage à l'ingéniosité d'Édouard et Jean Niermans, transformant «le problème acoustique en problème mécanique » par l'installation de l'orgue sur un chariot mobile se déplaçant sur des rails perpendiculaires au cadre de scène, afin de l'installer sur le plateau ou de le ranger en fond de scène dans le local prévu à cet effet, selon les besoins :

L'orgue est logé dans une sorte de boîte métallique ouverte sur le devant, et dont le plancher est constitué par une grande poutre roulant sur des rails placés au niveau de la scène. Quoique cet instrument gigantesque mesure 18 mètres de large, plus de 8 mètres de haut et qu'il pèse 75 tonnes, il pourra aisément être déplacé par un moteur de $7 \mathrm{CV}$ à une vitesse très faible, presque insensible pour le public, de 4 mètres à la minute.

Ainsi l'orgue peut se faire entendre soit en avant dans le cadre de scène (cas d'un récital ou de l'orchestre jouant avec orgue), soit en arrière (cas de l'exécution d'un opéra), soit dans toute position intermédiaire ; la console des claviers pouvant ellemême prendre vis-à-vis de l'instrument des positions quelconques, en avant sur le proscenium, sur la scène, dans la fosse d'orchestre, etc ${ }^{44}$.

La polyvalence de la nouvelle salle est donc l'occasion d'imaginer des solutions aux problèmes que posent ces usages multiples. La voix parlée n'est pas oubliée et les architectes Édouard et Jean Niermans, aidés de Jacques Brillouin, mettent en œuvre des traitements sur les parois et le plafond de manière à porter la voix en tous points de la salle. Car l'autre problématique de cette opération est celle de sa vastitude. La nouvelle salle doit accueillir 2800 spectateurs, les plus éloignés seront à 41 mètres du cadre de scène. Dans un article publié en 1937 par la revue Actualités scientifiques et industrielles, Jacques Brillouin insistait sur le problème des grandes salles et l'attention qu'elles requièrent pour le traitement acoustique. Cet article qui donne quelques règles générales sur le son et sa propagation dans l'espace est intéressant dans ses motivations, à savoir "d'être utile aux architectes ${ }^{45}$ ", constatant notamment que la plupart des ouvrages sur le sujet sont en anglais : «ils sont nombreux, et certains sont excellents. Mais ce sont des traités très techniques qui s'adressent beaucoup plus à l'ingénieur qu'à l'architecte ${ }^{46} \%$. L'intention de Jacques Brillouin n'est pas que les architectes se passent des services des acousticiens, mais qu'ils puissent maîtriser suffisamment les bases de cette science pour l'intégrer en amont, comme ils le font pour la mécanique entre autres. Si Brillouin constate que les architectes ont assez de connaissances pour envisager le calcul d'une charpente, «il n'en est pas de même en acoustique. Il en résulte pour l'acousticien consulté ensuite, la nécessité soit de se contenter de solutions bâtardes et médiocres, soit de demander de profondes modifications à un plan déjà présenté par l'architecte à son client. Situation évidemment fort délicate ${ }^{47} »$. A contrario, Jacques Brillouin constate et regrette les discours de techniciens ayant tendance "à négliger d'autres aspects du problème : à dire, "les formes importent peu; que l'architecte fasse sa salle, nous lui donnerons une 
acoustique convenable"48». Le constat d'un désintérêt ou du moins d'une méconnaissance réciproque motive donc le discours de cet ingénieur particulièrement soucieux de nouer le dialogue.

Les propos de Jacques Brillouin, de même que l'idée de créer ces chambres de réverbération pour une salle de concert accueillant une programmation éclectique, ainsi que certains choix scénographiques tels que la réduction de jauge par le déploiement d'une paroi mobile à l'aplomb du cadre de scène font de cette opération de 1937, pourtant tombée dans l'oubli ou mémorisée comme un échec, un objet d'étude très contemporain dans ses problématiques et ses solutions spatiales et techniques. Les plans dressés à la veille de la démolition de la salle en 1973 montrent sans équivoque que les chambres de réverbération avaient été alors transformées en dépôts de matériel son et lumière et de costumes. Il n'est pas certain qu'elles aient jamais servi à ce pour quoi elles étaient prévues. Ici se noue le dilemme de l'étude des lieux de spectacles: faut-il valoriser les qualités théoriques ou supposées de tel ou tel bâtiment, sans considérer les limites ou réserves que suscite leur exploitation réelle? La salle expérimentale de 1975 n'échappera pas, à son tour, aux critiques. À défaut d'être exemplaire, le site du Trocadéro peut se prévaloir d'avoir connu trois grandes salles de spectacle, monstrueuses sinon "ratées" selon les points de vue des usagers, particulièrement ambitieuses et innovantes quand on les met en perspective dans l'histoire de l'architecture théâtrale du xx ${ }^{\mathrm{e}}$ siècle.

[Lien au dossier pédagogique multimédia en ligne « Entendre le théâtre », à venir début juillet 2019]

\section{NOTES}

1. La mission principale du TNP est de créer et montrer à un vaste public des spectacles dramatiques. Il proposera aussi de nombreux concerts et des spectacles de danse.

2. Isabelle Gournay, Le Nouveau Trocadéro, Paris/Liège, IFA/Mardaga, 1985.

3. Pascal Ory, Le Palais de Chaillot, Arles, Actes Sud, 2006.

4. «Exposition internationale de Paris 1937. Arts et techniques dans la vie moderne, plan directeur ", novembre 1934, p. 6 ; cité par Isabelle Gournay, in Le Nouveau Trocadéro, op. cit., p. 26.

5. Ibid.

6. Programme $n^{\circ} 1$, document non daté, p. 2. A.N. F12/12420.

7. L'Écho de Paris, 11 décembre 1934.

8. Pascal Ory, Le Palais de Chaillot, op. cit, p. 31.

9. Programme $n^{\circ} 1$, op. cit., p. 1-2. A.N. F12/12420.

10. Programme $n^{\circ} 1$ bis, non daté, p. 4. A.N. F12/12420.

11. En effet, il est fréquemment écrit que les frères Niermans sont les auteurs de cette salle de spectacle. Or, fidèle aux recommandations du concours, le projet remis par eux en 1935 prévoit bien évidemment de conserver et transformer la salle des fêtes de 1878. Plusieurs plans datés de 1936 attestent l'implication du trio Carlu, Boileau et Azéma dans les choix préliminaires au dessin de la nouvelle salle, y compris son ornement. Nous y reviendrons dans une prochaine étude. 
12. Les plans de ce projet non réalisé sont conservés à la Bibliothèque de l'Hôtel de Ville de Paris. En 1886, Alphonse Gosset en publie une version, datée de 1875, dans son Traité de construction des théâtres (Paris, Baudry et $\mathrm{C}^{\mathrm{ie}}, \mathrm{pl}$. 21). La salle est alors baptisée « Opéra populaire ".

13. Dans son ouvrage, Pascal Ory explique (p. 54) que Davioud et Bourdais ont conçu l'acoustique de la salle des fêtes par « homologie visuelle", autrement dit, en assimilant la réflexion sonore à celle des rayons lumineux. Soucieux de la propagation du son dans une salle où les spectateurs les plus éloignés sont à 70 mètres de la scène, Davioud et Bourdais ont eu soin de placer des réflecteurs sur la scène. Cependant, le vrai problème de la salle des fêtes, ce sont les échos, ce que Raymond Cogniat commente avec ironie dans Le Monde illustré du 18 décembre 1937 évoquant « la triste et poussiéreuse salle dont l'acoustique imprévue répétait, en écho, au moins trois ou quatre fois chaque son ".

14. Lettre de Jules Bourdais au ministre de l'Instruction publique et des Beaux-Arts, 6 janvier 1903. AN F21/6145.

15. Ibid.

16. Ces détails sont fournis dans une lettre du 13 avril 1904, rédigée par le chef du Bureau des bâtiments civils. AN F21/6145. Précisons que Gabriel Davioud est décédé en 1881. Jules Bourdais (1835-1915) assurera donc le suivi des divers chantiers d'amélioration de la salle.

17. Ibid.

18. Ibid.

19. Ibid.

20. Les Archives nationales conservent à ce sujet un courrier de Gustave Lyon à Jules Bourdais, daté du 10 mai 1906, dans lequel l'acousticien laisse entendre son mécontentement devant l'attitude de Bourdais, réfractaire à ces travaux. Indépendamment du litige, Lyon y fournit quelques précisions sur les études qu'il a entreprises pour mesurer précisément cette conque dont il n'avait pas eu les plans, afin d'en épouser au mieux les courbes, tout en essayant d'améliorer l'absorption. A.N. F21/6145.

21. Ibid.

22. Fondée en 1807 par Ignace Pleyel auquel s'associe Auguste Wolff, cette entreprise de facture de pianos est confiée en 1883 à Gustave Lyon, musicien et ingénieur, gendre de Pleyel. Gustave Lyon utilisera les ressources de l'usine créée en 1865 pour développer les instruments et prototypes nécessaires à l'étude acoustique du Trocadéro. Il est aussi à l'origine de la salle Pleyel en 1925. Très intéressé par l'acoustique des salles, Gustave Lyon crée au sein de la Maison Pleyel un bureau d'études et de correction en orthophonie des salles. Un papier à en-tête de ce bureau en témoigne (A.N. F21/6145).

23. A.N. F21/6145.

24. Cette première phase d'étude conduit à un litige avec Jules Bourdais critiquant la solution préconisée, ceci amenant au retrait de Lyon quant à la réalisation définitive des panneaux de la conque.

25. Lettre de Gustave Lyon au directeur (des Beaux-Arts), 15 mai 1912. A.N. F21/6145.

26. Minute de Lettre du secrétaire d'État aux Beaux-Arts à Jules Bourdais, 7 juillet 1911. A.N. F21/6145.

27. B.N. Postma, S. Dubouilh, B. F. G. Katz, «An archeoacoustic study of the history of the Palais du Trocadéro (1878-1937) », The Journal of the Acoustical Society of America, vol. 145, n 4, 2019.

28. Les discussions budgétaires du 26 décembre 1903 relatent à ce sujet l'intervention du rapporteur rappelant que « depuis de longues années, on cherche à organiser, à Paris, le théâtre populaire ». La salle des fêtes du Trocadéro, dotée de 5000 places, semble alors adéquate pour "donner aux légitimes aspirations du peuple, la satisfaction artistique qu'il est en droit d'attendre ». A.N. F21/6145.

29. Cité par Chantal Meyer-Plantureux in Théâtre populaire. Enjeux politiques, de Jaurès à Malraux, Bruxelles, Éditions Complexe, 2006, p. 137. 
30. Programme, non daté. A.N. F12/12420.

31. En effet, il n'y a aucun doute sur le rôle de Jacques Brillouin dans ce projet de 1937, mais il n'existe aucune trace de contrat ou document le citant ou le nommant, ni dans les fonds des Archives nationales, ni dans le fonds Jacques Carlu de l'Institut français d'architecture.

32. Émile Fleurent, Lettre au Commissaire général de l'Exposition universelle, 22 juillet 1935. A.N. F12/12976 (souligné dans le texte).

33. Émile Fleurent, Lettre au Commissaire général de l'Exposition universelle, $1^{\mathrm{er}}$ octobre 1935. A.N. F12/12976.

34. Comptes-rendus hebdomadaires des séances de l'Académie des sciences, séance du 21 mars 1938, p. 897.

35. Robert Fleurent, L'Insonorisation des bureaux, Paris, Institut technique des administrations publiques (ITAP), 1955. Conférence donnée le 25 mai 1954 lors de la Journée d'études sur les facteurs d'ambiance dans les bureaux, organisée par l'ITAP.

36. Ces deux projets ont été réalisés par les Ateliers Jean Nouvel associés à Brigitte Métra. Voir supra Brigitte Métra, "Architecture et acoustique des salles", entretien réalisé par Sandrine Dubouilh.

37. Charles-Edmond Sée, «Les aménagements du nouveau Théâtre du Trocadéro ", Le Génie civil, $n^{\circ} 2910,21$ mai 1938, p. 425-430.

38. Jacques Brillouin, "La salle de spectacle du nouveau Trocadéro », La Revue musicale, juinjuillet 1937. Dossier « L'exposition et la musique », p. 5.

39. Ibid.

40. Ibid.

41. Cette commission est composée de Joseph Bonnet (Saint-Eustache), Marcel Dupré (SaintSulpice), Édouard Mignan (La Madeleine), Ludovic Panel (Sacré-Cœur), Charles Tournemire (Sainte-Clotilde). Les documents relatifs aux transformations de l'orgue sont consultables aux Archives nationales, 19960511/3.

42. A.N. 19960511/3.

43. Ibid.

44. Fernand Gonzales, "L'orgue monumental du palais de Chaillot », La Revue musicale, op. cit., p. 8 .

45. Jacques Brillouin, "Acoustique du bâtiment I, l'acoustique et la construction, bases de la technique à l'usage des architectes, décorateurs, entrepreneurs, etc. », Actualités scientifiques et industrielles, $\mathrm{n}^{\circ} 457,1937$, p. 3.

46. Ibid.

47. Ibid., p. 4.

48. Ibid., p. 6.

\section{RÉSUMÉS}

Étudié dans le cadre du programme de recherche ECHO, le Palais du Trocadéro s'est révélé être un objet très intéressant pour les recherches sur l'histoire de l'acoustique des salles en France. Construit à l'occasion de l'Exposition universelle de 1878 sur les plans de Davioud et Bourdais, il abrite une grande salle des fêtes de 5000 places. Son exploitation posera de nombreux problèmes, notamment acoustiques, motivant les recherches de Gustave Lyon qui y réalisera des 
mesures et tentera des corrections. Lorsque la décision de démolir la salle des fêtes est prise pour l'Exposition universelle de 1937, une attention particulière est portée à l'acoustique de la nouvelle salle de spectacle. Ses dimensions mais aussi sa vocation polyvalente justifient le soin apporté au son: l'acousticien Jacques Brillouin innove en préconisant des chambres de réverbération.

Studied as part of the ECHO research program, the Palais du Trocadéro revealed itself to be a highly interesting subject for a research project on the history of room acoustics in France. Built for the 1878 Exposition universelle according to the plans of Davioud and Bourdais, the Palais du Trocadéro housed a large concert hall of 5000 seats. Its operation would pose numerous problems, notably in regard to acoustics, thus motivating research by Gustave Lyon, who implemented measures and attempted corrections. When the decision was made to demolish the hall for the 1937 Exposition universelle, the acoustics of the new concert hall were given special attention. Both the hall's dimensions and versatility justified the care accorded to sound, which was enhanced by the acoustician Jacques Brillouin through his innovative recommendation of echo chambers.

\section{INDEX}

Mots-clés : Palais du Trocadéro, acoustique des salles, histoire architecturale des théâtres, chambres de réverbération

Keywords : Palais du Trocadéro, room acoustics, history of theatrical architecture, echo chambers

\section{AUTEUR}

\section{SANDRINE DUBOUILH}

Architecte DPLG, professeure des universités, Sandrine Dubouilh enseigne à l'université Bordeaux Montaigne. Elle est spécialiste de l'histoire de la scénographie et de l'architecture théâtrale ( $\mathrm{XIX}^{\mathrm{e}}-\mathrm{XX}^{\mathrm{e}}$ siècles). Ses recherches actuelles portent sur l'histoire des salles de spectacle du Trocadéro et sur l'acoustique des salles (programme de recherche ECHO), ainsi que sur les lieux de spectacle et divertissement à Bordeaux (XVIII ${ }^{\mathrm{e}}-\mathrm{XXI}{ }^{\mathrm{e}}$ siècles), en collaboration avec Pauline Beaucé.

Parmi ses publications :

Une architecture pour le théâtre populaire, 1870-1970, Nantes, Actualité de la scénographie,

«Scéno+ », 2012.

"Quelle place pour le son dans la conception des lieux de théâtre ? », in J.-M. Larrue et M.M. Mervant-Roux (dir.), Le Son du théâtre (XIXe-XXIe siècle), Paris, CNRS Éditions, 2016, p. 117-131. «Écrire une histoire de l'écoute à travers l'architecture des lieux de spectacle », in H. BouvierSmith et M. Chénetier-Alev (dir.), L'Écho du théâtre. Dynamiques et construction de la mémoire phonique, XX'e-XXI siècles, Revue Sciences/Lettres [en ligne], $\mathrm{n}^{\circ}$ 5, Rue d'Ulm, 2017.

« Du cirque au cinéma : jalons pour une histoire des lieux de spectacles rue Castelnau d'Auros à Bordeaux (1835-1898) » (avec P. Beaucé), in F. Fix (dir.), Théâtre et ville, Dijon, EUD, 2018, p. 141-152.

« La Maison de la Culture au cœur des utopies sociale, urbaine et scénographique (1965-1968) », 
in A. Folco (dir.), Maison de la Culture de Grenoble. 1968 : un édifice, des utopies, Revue d'histoire du théâtre, $\mathrm{n}^{\circ}$ 279, 2018, p. 63-78.. 